\title{
Vaccine Provider
}

National Cancer Institute

\section{Source}

National Cancer Institute. Vaccine Provider. NCI Thesaurus. Code C102430.

A person or organization that is licensed, certified or otherwise authorized or permitted

by law to administer vaccinations in the ordinary course of business or practice of a profession. 